\title{
MINIREVIEW
}

\section{Bunched and Madm: a novel growth-regulatory complex?}

\author{
Kieran F Harvey* \\ See research article at http://jbiol.com/content/9/1/9
}

\begin{abstract}
By combining Drosophila genetics and proteomics Gluderer et al. report in this issue of Journal of Biology the isolation of a novel growth-regulatory complex consisting of Bunched and Madm. Future study of this complex will address the precise mechanism of growth control, regulation of complex activity, the interface with other growth pathways and a potential role in human cancer.
\end{abstract}

Cancer is a complex group of diseases that stems from the accumulation of mutations in genes that control fundamental biological processes, such as cell growth, proliferation, survival and migration. The study of model organisms has provided several seminal contributions to our understanding of cancer, originating with Theodor Boveri's hypothesis drawn from sea urchin-based studies in the early 1900s that cancer arises because of chromosomal abnormalities [1]. In more recent times, mutations in dozens of genes have been shown to contribute to the genesis of different cancers and their discovery has been facilitated by various approaches: isolation of oncogenic viruses; close examination of familial cancer; cytogenetics; positional cloning; genetic screens in model organisms followed by analysis of mutation status of human homologs in cancerous cells; genome-wide analysis of gene expression and copy number changes; and high-throughput sequencing and genome-wide association studies.

\section{Flies and cancer}

Drosophila melanogaster has proved to be a very valuable organism for the discovery of novel signaling proteins

*Correspondence: kieran.harvey@petermac.org

Cell Growth and Proliferation Laboratory, Peter MacCallum Cancer Centre,

7 St Andrews Place, East Melbourne, Victoria 3002, Australia and Department of

Pathology, University of Melbourne, Parkville, Victoria 3010, Australia and pathways that control various biological processes. A major tool in the armory of Drosophila biologists has been forward genetic screens. Over time, many different mutation and screening strategies have been used by Drosophila biologists, with great success. One such screening approach, the so-called Flp recombinase/Flp recombinase target (Flp/FRT) clonal screen, has facilitated the discovery of many new growth regulators by several laboratories, predominantly in the late 1990s and early 2000s. Essentially, the Flp/FRT technique allows the creation of patches of homozygous mutant tissue in a heterozygous animal. Thus, growth-regulatory genes that normally cause pre-adult lethality when homozygous were recovered using such screens.

Subsequently, many of the human homologs of genes that regulate growth in Drosophila were linked to the genesis of human cancers. These included several regulators of the insulin and Target of rapamycin (TOR) pathways [2], the ubiquitin ligase Archipelago (Ago) [3] and a novel signaling pathway, the Salvador/Warts/Hippo $(\mathrm{SWH})$ pathway $[4,5]$. Deregulation of the insulin and TOR pathways has well described links to human cancer; for example, PTEN, which represses activity of the insulin pathway, is one of the most commonly mutated human tumor suppressor genes. Following its discovery in 2001, the human ortholog of Ago has been shown to be mutated at varying frequencies in a wide range of human cancers, including ovarian cancer, colon cancer and $\mathrm{T}$ cell acute lymphoblastic leukemia [6]. Several lines of evidence have also linked altered activity of different SWH pathway components to human cancer, although the degree to which deregulation of this pathway contributes to the genesis of different human cancers is still being determined [5].

\section{Bunched and Madm: a new growth-promoting complex}

Most Drosophila Flp/FRT screens were designed to isolate genes that restrict tissue growth. The screen originally used by the Hafen laboratory [2] was designed in such a way that it could capture both growth 
suppressors and genes required to promote growth. Two such growth-promoting genes were bunched (bun) and Mlf1 adapter molecule (Madm), which were isolated based on the fact that tissue harbouring mutations in these genes exhibited a growth deficiency [7,8]. The mechanism of action and function of neither gene has been well characterized in flies or mammals. Bun is a putative transcription factor that influences Drosophila oogenesis and nervous system development. There are four mammalian bun homologs, one of which, Transforming growth factor- $\beta$-stimulated clone 22 (TSC22D1), has been hypothesized to be a tumor suppressor gene from its ability to repress growth and survival of cultured cells [9]. However, its precise function, and that of its three homologs, has not been well characterized in vivo in mammals. The bun gene encodes variant gene products, and only the long Bun proteins (such as BunA) seem to promote growth, whereas shorter isoforms have been shown to antagonize longer Bun isoforms $[7,10]$. Although Madm was first isolated using its ability to bind the Myeloid leukemia factor 1 (Mlf1) oncoprotein [11], the work by Gluderer et al. in this issue of Journal of Biology [8] is the first in vivo functional study of Madm.

The findings of Gluderer et al. [8] suggest that Bun and Madm form a novel growth-controlling complex (Figure 1). Their work [8] highlights the power of combining multiple experimental approaches (in this case genetics and biochemistry) to elucidate protein function. Madm was isolated as a BunA-interacting partner by affinity purification and mass spectrometry, and, in parallel, it was isolated in the above-described Flp/FRT growth screen. The combined approach provided complementary validation for the function of Madm and also provided a hint at mechanism: that Bun and Madm control growth tissue growth in partnership. They appear to do so in an obligate fashion as overexpression of both bun and $M a d m$ is required to induce tissue overgrowth [8]. The major challenges arising from this discovery are to define the mechanism by which BunA and Madm promote growth and to explore the role of their mammalian homologs in normal tissue growth and in cancer.

\section{Possible mechanisms of action of the BunA-Madm complex}

Gluderer et al. have proposed a number of possible mechanisms by which BunA and Madm promote tissue growth [8]. One possibility is that BunA and Madm are components of an existing growth-regulatory pathway. Tissues lacking bun or Madm show some similarities to the phenotypic characteristics of tissues with compromised insulin or TOR pathway activity, such as small cell size and reduced cell number $[7,8]$. However, Gluderer et al. previously showed [7] that bun is unlikely to control insulin or TOR pathway activity. Alternatively, BunA and

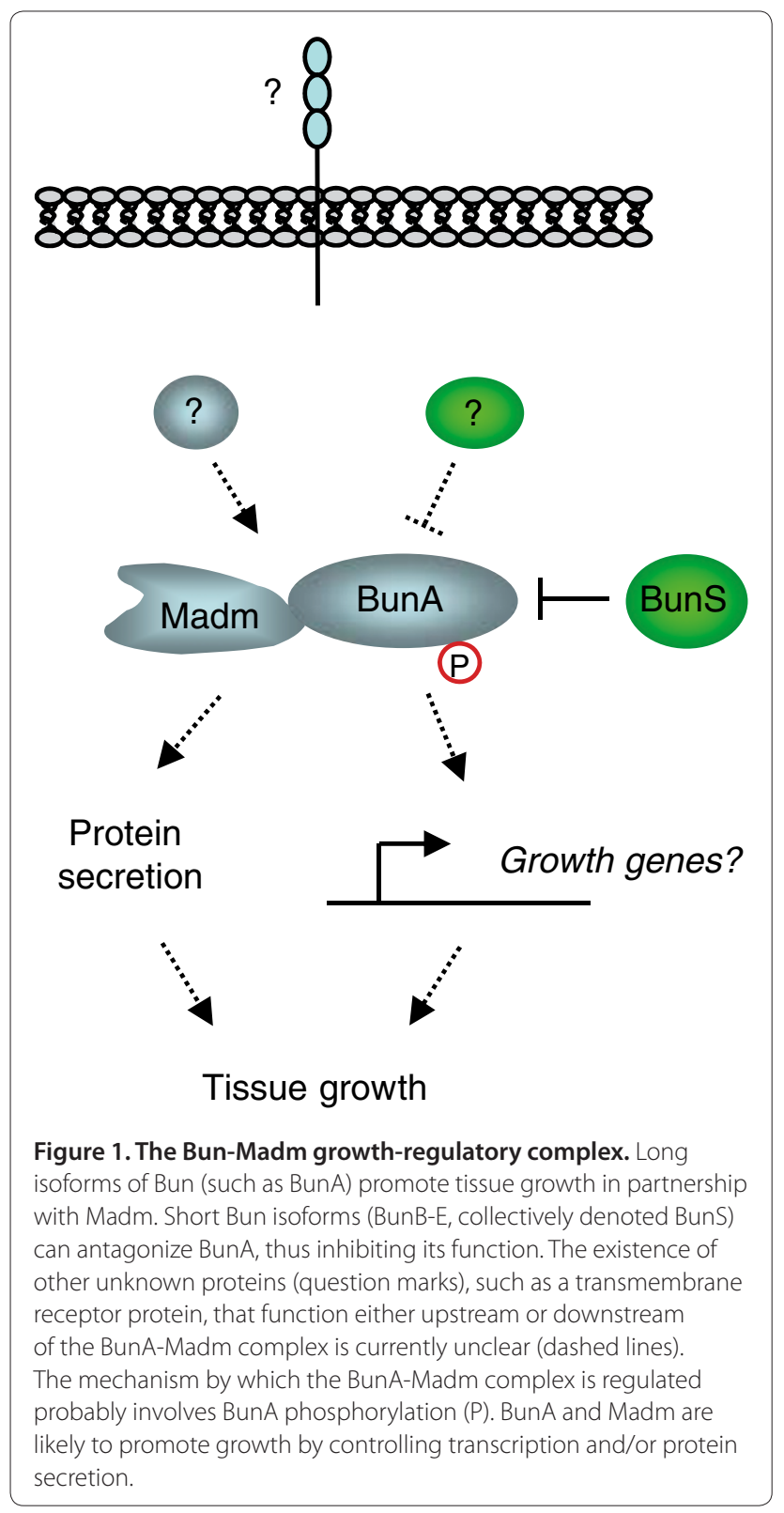

Madm might constitute a novel growth-promoting pathway. If this is the case, an obvious question is how a Bun/ Madm pathway would interface with other growth pathways to coordinately modulate tissue growth, particularly during development, when patterning cues and nutrient signals need to be orchestrated to specify organ and organism size.

The exact molecular steps by which BunA and Madm promote tissue growth also remain mysterious. Bun has been proposed to be a transcription factor and therefore might stimulate expression of a growth-promoting transcriptional program, but so far no such target genes have been identified. Loss-of-function mutations in bun were 
recovered lacking the carboxyl terminus, which includes the DNA-binding TSC box of BunA and the leucine zipper [7]. To establish whether BunA-mediated transcription is essential for BunA-Madm to drive growth, it would be desirable to determine whether a BunA protein that is incapable of binding DNA as a result of TSC-box missense mutations that abrogate DNA binding can rescue bun deficiency.

Another possibility is that the BunA-Madm complex regulates protein secretion. BunA and Madm both localize to the Golgi, and Madm has been shown to affect transport from the endoplasmic reticulum to Golgi $[8,12]$. In addition, RNAi-mediated repression of either Madm or bun was found to inhibit protein secretion in cultured cells [13], although it is unclear whether BunA and Madm regulate secretion in a cooperative manner. It is also unclear whether the ability of BunA and Madm to control protein secretion is linked to their role as regulators of tissue growth.

\section{Regulation of the Bun-Madm complex}

The regulation of BunA and Madm is another important outstanding issue that will need to be addressed in future studies. For example, it will be important to identify the proteins that function upstream and downstream of BunA-Madm, as well any potential transmembrane receptor proteins regulating their activity (Figure 1). A common feature of the regulation of all known signaling pathways is phosphorylation. BunA is phosphorylated in a conserved amino-terminal domain [14] and this is likely to modulate its activity. Identification of kinases and phosphatases that regulate BunA, and examination of a role for Madm in mediating BunA phosphorylation, should shed light on the regulation of growth by these proteins. Madm has been postulated to regulate the activity of Mlf1 by controlling its nuclear access [11] and may exert similar control over BunA. However, so far no evidence of nuclear localization of BunA has been found [8].

Transcriptional control of the bun gene offers another potential mode of regulation of the BunA-Madm complex. In cultured mammalian cells, TSC22D1 transcription is controlled by TGF $\beta$ [15], but it is unclear whether this mode of transcriptional control is conserved for bun genes across diverse species. In addition, as described by Gluderer et al. [8], the bun locus gives rise to long and short Bun proteins and the short Bun proteins have been shown to antagonize the longer isoforms, possibly by heterodimerization and subsequent disruption of growth-promoting BunA complexes [7]. Modulation of bun transcription under utilization of different promoters that yield discrete Bun isoforms might therefore modulate the net growth-regulatory output of the bun locus.

\section{Mammalian Bun and Madm homologs and cancer}

There is a high degree of conservation between growth control programs that function in flies and in mammals. A major unresolved question regarding the Bun-Madm growth control complex is whether deregulation of their human homologs contributes to the genesis of cancer. The short isoform of TSC22D1 was initially hypothesized to be a tumor suppressor [9], but the growth-regulatory role of long TSC-22 isoforms and of Madm in mammals remains unresolved. Assuming that the function of BunA and Madm is conserved between flies and mammals, the loss of Madm or of one of the four TSC22 family genes might be expected to impair tissue growth and therefore not to be tumor-promoting. In addition, given that genetic studies in Drosophila have shown that BunA and Madm promote growth in an obligate partnership, human homologs of both genes would need to be overexpressed or amplified in order to promote tumorigenesis, which is unlikely to be a common event. The most likely tumorpromoting effect of this complex might be reduced expression of short isoforms of the TSC22 gene family, which might alleviate TSC-22-Madm repression. While these possibilities are being investigated in mammalian cells, studies in Drosophila are likely to continue to lead the way in unraveling the mechanism by which the newly identified Bun-Madm complex controls tissue growth.

Published: 11 February 2010

\section{References}

1. Boveri T: Über mehrpolige Mitosen als Mittel zur Analyse des Zellkerns. [Concerning multipolar mitoses as a means of analysing the cell nucleus] Verh Phys Med Ges Würzburg 1902, 35:67-90.

2. Hafen E: Cancer, type 2 diabetes, and ageing: news from flies and worms. Swiss Med Wkly 2004, 134:711-719.

3. Moberg KH, Bell DW, Wahrer DC, Haber DA, Hariharan IK: Archipelago regulates Cyclin E levels in Drosophila and is mutated in human cancer cell lines. Nature 2001, 413:311-316.

4. Tapon N, Harvey KF, Bell DW, Wahrer DC, Schiripo TA, Haber DA, Hariharan IK: Salvador promotes both cell cycle exit and apoptosis in Drosophila and is mutated in human cancer cell lines. Cell 2002, 110:467-478.

5. Harvey K, Tapon N: The Salvador-Warts-Hippo pathway - an emerging tumour suppressor network. Nat Rev Cancer 2007, 7:182-191.

6. Welcker M, Clurman B: FBW7 ubiquitin ligase: a tumour suppressor at the crossroads of cell division, growth and differentiation. Nat Rev Cancer 2008, 8:83-93.

7. Gluderer S, Oldham S, Rintelen F, Sulzer A, Schutt C, Wu X, Raftery LA, Hafen E, Stocker $\mathrm{H}$ : Bunched, the Drosophila homolog of the mammalian tumor suppressor TSC-22, promotes cellular growth. BMC Dev Bio/ 2008, 8:10.

8. Gluderer S, Brunner E, Rintelen F, Germann, M, Jovaisaite V, Changqing L, Rentsch CA, Hafen E, Stocker H: Madm (MIf1 adapter molecule) co-operates with Bunched A to promote growth in Drosophila. J Biol 2010, 9:9.

9. Nakashiro K, Kawamata H, Hino S, Uchida D, Miwa Y, Hamano H, Omotehara F, Yoshida H, Sato M: Down-regulation of TSC-22 (transforming growth factor beta-stimulated clone 22) markedly enhances the growth of a human salivary gland cancer cell line in vitro and in vivo. Cancer Res 1998, 58:549-555.

10. Wu X, Yamada-Mabuchi M, Morris EJ, Tanwar PS, Dobens L, Gluderer S, Khan S, Cao J, Stocker H, Hafen E, Dyson NJ, Raftery LA: The Drosophila homolog of human tumor suppressor TSC-22 promotes cellular growth, proliferation, and survival. Proc Natl Acad Sci USA 2008, 105:5414-5419.

11. Lim R, Winteringham LN, Williams JH, McCulloch RK, Ingley E, Tiao JY, Lalonde JP, Tsai S, Tilbrook PA, Sun Y, Wu X, Morris SW, Klinken SP: MADM, a novel 
adaptor protein that mediates phosphorylation of the 14-3-3 binding site of myeloid leukemia factor 1. J Biol Chem 2002, 277:40997-41008.

12. De Langhe S, Haataja L, Senadheera D, Groffen J, Heisterkamp N: Interaction of the small GTPase Rac3 with NRBP, a protein with a kinase-homology domain. Int J Mol Med 2002, 9:451-459.

13. Bard F, Casano L, Mallabiabarrena A, Wallace E, Saito K, Kitayama H, Guizzunt G, Hu Y, Wendler F, Dasgupta R, Perrimon N, Malhotra V: Functional genomics reveals genes involved in protein secretion and Golgi organization. Nature 2006, 439:604-607.

14. Bodenmiller B, Malmstrom J, Gerrits B, Campbell D, Lam H, Schmidt A, Rinner O, Mueller LN, Shannon PT, Pedrioli PG, Panse C, Lee HK, Schlapbach R, Aebersold R: PhosphoPep--a phosphoproteome resource for systems biology research in Drosophila Kc167 cells. Mol Syst Biol 2007, 3:139.

15. Shibanuma M, Kuroki T, Nose K: Isolation of a gene encoding a putative leucine zipper structure that is induced by transforming growth factor beta 1 and other growth factors. J Biol Chem 1992, 267:10219-10224.

doi:10.1186/jbiol219

Cite this article as: Harvey KF: Bunched and Madm: a novel growthregulatory complex? Journal of Biology 2010, 9:8. 